\title{
When do we stop digging? Conditions on a fundamental theory of physics
}

\author{
Karen Crowther ${ }^{* \dagger}$
}

\begin{abstract}
In seeking an answer to the question of what it means for a theory to be fundamental, it is enlightening to ask why the current best theories of physics are not generally believed to be fundamental. This reveals a set of conditions that a theory of physics must satisfy in order to be considered fundamental. Physics aspires to describe ever deeper levels of reality, which may be without end. Ultimately, at any stage we may not be able to tell whether we've reached rock bottom, or even if there is a base levelnevertheless, I draft a checklist to help us identify when to stop digging, in the case where we may have reached a candidate for a final theory. Given that the list isaccording to (current) mainstream belief in high-energy physics - complete, and each criterion well-motivated, I argue that a physical theory that satisfies all the criteria can be assumed to be fundamental in the absence of evidence to the contrary.
\end{abstract}

\section{Introduction}

It may come as a surprise, but not one of our current theories of physics are generally considered fundamental. This is in spite of the fact that the standard model of particle physics (our best theory of matter), and general relativity (our best theory of space and time), are the most accurate and successful scientific theories ever! Physicists are not content to rest with these theories; they expect more-fundamental physics to lie beyond - to be buried deeper underneath.

In this essay, I consider why physicists are apparently so hard to satisfy - that is, I ask why these two theories are not thought to be fundamental. The aspects of these theories that are responsible for their reputation as non-fundamental can then be inverted, and framed as conditions that any theory must satisfy if it is to be considered fundamental. Underlying these conditions, I discover, are two general principles, which can be used to motivate some further conditions. In this way, I compile a checklist of necessary conditions on a fundamental theory, according to common belief in high-energy physics (also known as particle physics). These conditions - although they are not all able to be precisely defined, nor rigorously justified - reflect the essential character of physics itself. Thus, given that the list is - from the perspective of current physics - complete, and each criterion well-motivated, I argue that

*Department of Philosophy, University of Geneva

${ }^{\dagger}$ karen.crowther@unige.ch 
a theory of physics that satisfies all the criteria can be assumed to be fundamental in the absence of evidence to the contrary.

The structure of the essay is as follows: In $\S 2$, I outline some of the different ideas of fundamentality associated with modern physics, before, in $\S 3$, explaining why neither the standard model of particle physics nor general relativity (GR) are typically considered fundamental. Following this, $\S 4$ presents the necessary conditions that physicists apparently place on a fundamental theory and explores what these reveal about the nature of physics, as well as its dream of a final theory. In $\S 5$, I consider the implications of these conditions for quantum gravity (QG) - the (currently unknown) theory that is supposed to be more fundamental than GR and quantum field theory. And, conversely, I also investigate what QG reveals about our conception of fundamentality.

\section{Fundamental and more-fundamental in modern physics}

Conceptions of fundamentality in modern physics have been heavily shaped by the framework of effective field theory (EFT), and its associated philosophy. The framework of EFT is a means of constructing theories - called effective field theories - that are each valid only at a given "level", i.e., at large distances (corresponding to low energy scales, since energy is inversely proportional to length) compared to a particular short length (high-energy) scale "cutoff", $\Lambda$. To do this, we can start either with a short-distance theory and then use the toolbox of EFT to produce a theory that describes the same system at larger distances, or the other way around (from a large-distance theory to one that describes the system at shorter-length scales), or we can start from scratch to construct a theory at the required scale when no shorter- or longer-distance one is available. Effective theories are generally not considered to be fundamental, because they break down (i.e., cease to be predictive) at lengths approaching $\Lambda$. The picture presented by this framework is of a "tower of theories", each valid at a different level. Each theory is framed in terms of the appropriate parameters for its particular level, representing the physical interactions that are important at that level, and these parameters are specific to each theory - they do not exist at the levels above or below in the tower [1].

Because of this, there is a compelling case to be made that what is fundamental depends on the level we are interested in - for each level there is a theory that clearly describes the relevant physics of the system being studied, and is framed in terms of the appropriate parameters [2]. While physicists generally believe that we could in principle use a shorter length scale theory in order to make predictions about the system at some particular larger distance scale (i.e., if we had access to the required computational resources, plus the ability to use them), to do so would not only be very (and needlessly) complicated, but would also hopelessly obscure the picture of the relevant physics at the scale of interest. We do not need to use a theory of atoms in order to describe a game of baseball, for instance. And yet, there is an asymmetry between the levels - we believe that the laws we use to describe the motion of the baseball after being hit depend on those of atomic physics, but not that the laws of atomic physics depend on the laws used to describe the baseball. Atomic theory is thought to

be more fundamental than the laws we use to predict the trajectory of a baseball - the atoms and their interactions are "more basic" than any macroscopic objects that they compose. 
This asymmetry is captured in the way we move between theories: It is generally believed that in principle, with full knowledge of the physics of a system at a particular short-length scale (plus, again, the required computational resources and the ability to use them), we could arrive at results valid at any larger scales without requiring any additional information. On the other hand, the large-scale physics is supposed to underdetermine the shorter-scale theory: We could not, even in principle, derive the correct theory of a system at smalllength scales from a complete description of its physics at some larger length scale. More information would be required. For this reason, the tower of theories is usually thought to be ordered hierarchically, with the shorter-scale theories being more fundamental, and so lower on the tower, than the larger-scale, "higher-level" ones. ${ }^{1}$ I use this notion of relative fundamentality here. ${ }^{2}$

We encounter problems, however, when we attempt to move from relative to absolute terms - what does it mean for a theory to be fundamental rather than just more- or lessfundamental? How do we define "rock bottom" of the tower? We might suppose it would be a theory valid at the tiniest length scales. Yet, according to quantum field theory (QFT) the framework within which the standard model of particle physics is formulated (and, as I explain below, is also understood in terms of EFT) - there is an arbitrarily large amount of energy available in the vacuum, and so the tower may be endless, "shorter and shorterlength turtles all the way down", thus implying that there is no final theory [3]. Commonly, it is thought that the way to escape this conclusion is to recognise, though, that we are not trapped in the framework of QFT $[4,5,6]$. It is possible that we find a theory that is not a $\mathrm{QFT}^{3}$, and which yields predictions at the tiniest length scales.

Although stepping outside the framework of QFT may free us from worrying about an arbitrarily large amount of energy available in the vacuum, ${ }^{4}$ however, it does not save us from the possibility that there is still new physics beyond any theory that we arrive at. The recognition that we are not trapped in the framework of any given theory produces an epistemic worry: Even if we reach a theory that yields predictions for the tiniest length scales (or, equivalently, all possible high-energy scales), we cannot be sure that these predictions are actually correct - unless, of course, we have access to experimental data at all possible high energy scales!

A theory (whether a QFT or not) that is formally predictive at all possible high energy scales is said to be $U V$ complete ("UV" referring to the short-wavelength end of the electromagnetic spectrum, the ultraviolet). Although I take it that a theory being UV complete is

\footnotetext{
${ }^{1}$ While this discussion reflects the beliefs of contemporary high-energy physics, I must register my own scepticism regarding such a "reductionist" picture, and refer to the substantial literature on emergence in science. Particularly, I have doubts about the basis of these "in principle" claims, and their meaningfulness.

2 There is another common, yet distinct notion of relative fundamentality in physics that is levelindependent, and associated with more general theories, rather than higher-energy theories. I do not discuss this conception here. As we shall see, however, equating "more fundamental" with "shorter-distance", is problematic because the very idea of distance may cease to be applicable at some point, and yet we may have reasons to expect there to be another, presumably more fundamental, theory beyond that point-i.e., beyond the domain of space and time - which could then not be called a "shorter-distance" theory!

${ }^{3}$ Indeed, as I discuss below, QFT is not considered a fundamental framework, and so it is expected that a fundamental theory will not be a QFT.

${ }^{4}$ This arbitrarily large vacuum energy may, in fact, be interpreted as an artifact of a non-fundamental formalism (§3.1).
} 
necessary for its being fundamental, ${ }^{5}$ being UV complete is not sufficient for a theory to be fundamental - it does not guarantee that there is "nothing beyond" [7]. For example, consider Newton's laws of motion: These are formally predictive in all domains, yielding results that are prima facie mathematically sensible. Yet we know that these laws are not correct at all scales: At small length scales (and under particular conditions) they must be replaced by quantum-mechanical laws, and for large velocities they are replaced by relativistic laws. Another example is quantum chromodynamics (QCD, the theory of the strong nuclear force), which is UV complete and yet, as I discuss below, is not considered fundamental. ${ }^{6}$

So, a theory formally being predictive to all high-energy scales, and thus apparently being the lowest brick in the tower (or, at least, one of the bricks at the lowest level of the tower), is no guarantee that it is in fact a fundamental theory-UV completeness alone is not enough reason to stop digging. Yet, it is one constraint on a fundamental theory. ${ }^{7}$ In order to understand what kind of theory would motivate physicists to stop digging, and to answer the question of what it means for a theory to be fundamental, I now invert it: Why do we not consider our current best theories of physics to be the final word? Why are we currently digging for a more fundamental theory?

\section{Why our current best theories of physics are not fundamental}

\subsection{Quantum field theory and the standard model}

We must distinguish between the framework of QFT, and particular QFTs (i.e., the theories formulated within this framework). To begin with, consider the framework of QFT; there are three inter-related reasons it is not considered fundamental. Firstly, the framework is mathematically ill-defined, which means, secondly, that, traditionally, ${ }^{8}$ the theories it generates are plagued by singularities - infinities pop up all over the place (e.g., in the arbitrarily large vacuum energy mentioned above). Some of these need to be dealt with in order to render the afflicted theories usable, and the procedure by which this is done is known as renormalisation.

The third reason the framework is not considered fundamental is that, although it utilises special relativity, it is not generally relativistic - it does not take into account our best theory of spacetime. Many physicists interpret this as the cause of the ill-definedness of the framework, as well as the singularities that appear within its theories. The modern interpretation of QFT holds that these singularities are artifacts of a non-fundamental framework, and that their appearance in our QFTs is the result of our ignorance of the more-fundamental

\footnotetext{
${ }^{5}$ If this were not true, then it would mean that the world is just not amenable to scientific description at extremely high-energy scales. But my arguments in $§ 4$ justify our dismissal of this "UV silence scenario" [7].

${ }^{6}$ Physicists usually distinguish between a fundamental theory and a final theory, arguing that although QCD is not a final theory, its UV completeness means that it is a fundamental theory. On this reasoning, Newtonian mechanics would also be considered a fundamental, though not final, theory. I argue below $\S 4$ that this reasoning is not consistent with the rest of the conditions on a fundamental theory.

${ }^{7}$ Neglecting the possibility of the UV silence scenario, Footnote 5. Also, I take a "theory without distance" (as in Footnote 2) to be UV complete, in the sense that it does not break down at any short distance scale.

${ }^{8}$ As we shall see, this problem was solved by considering QFT as EFT.
} 
physics at shorter-length scales beyond-physics that includes a quantum theory of gravity (discussed below). Thus, QFT is treated as an effective framework; i.e., as EFT.

Apart from being products of a non-fundamental framework, there are five reasons why QFTs are considered non-fundamental. (1) The first regards the necessity of renormalisation (for those theories where it is necessary) - the idea is, again, that the singularities dealt with by this procedure are due to our neglect of some shorter-scale (more fundamental) physics beyond. (2) Some theories are believed to be non-fundamental because they are not UVcomplete. Such theories, like quantum electrodynamics (QED, the quantum field theory of electromagnetism, describing light and the interactions of charged particles) break down at some short-distance scale, and are thus EFTs. ${ }^{9}$ (3) Many QFTs are supposedly nonfundamental because they are not exactly solvable (this is due to the ill-definedness of the framework), and so employ the approximation techniques of perturbation theory.

(4) Some QFTs are considered unnatural in a technical sense, that the large-distance theory sensitively depends on the choice of parameters in the more-fundamental (higher energy-scale) theory. This means that, if the high energy parameters had been the slightest bit different from their actual values, the large distance physics would depart radically from what we observe. If there is such a sensitive dependence on their values, the parameters appear to have been fine tuned: Standing out - to the physicists' eye - as "unnatural" and in need of explanation $[8,9,10]$. In practice, this idea of naturalness is seen as being satisfied when a theory does not contain dimensionless numbers that are either very large or very small. The standard model of particle physics is unnatural in this sense, due to one parameter: The Higgs particle mass. Physicists typically interpret this as meaning that if the Higgs particle mass had been slightly different at high energies, then our universe would likely not exist as we know it. ${ }^{10}$ The unnaturalness of the Higgs is thus thought to require explanation.

(5) Finally, the standard model of particle physics itself is non-unified. Although the standard model can be written as a single theory, it appears as a disjointed amalgam of separate (particle) fields, which drives many physicists to seek a more unified theory beyond [13]. This means that QFTs such as QCD that do not suffer any of the difficulties (1-3) at short-distances are still regarded as non-fundamental-not just because they are products of a non-fundamental framework, but because they are not part of a unified theory.

\subsection{General relativity}

The reasons for not believing GR fundamental are the motivations for seeking quantum gravity (QG) - the as-yet-undiscovered theory needed to describe physics in the domains where both QFT and GR are thought to be necessary. These domains include, for instance, the Planck scale, which is the unfathomably small distance of $10^{-32} \mathrm{~cm}$. The theory is expected to replace GR, and describe the more-fundamental physics that "underlies" spacetime. Since QG is supposed to be a quantum theory of spacetime (i.e., a theory that takes into account both quantum theory and GR) one of its motivations is the desire for unification, as well as a desire for a single theory, rather than multiple frameworks. Another factor driving the

\footnotetext{
${ }^{9}$ In the case of QED, this is due to the presence of a Landau pole divergence.

${ }^{10}$ There is growing dissent against the principle of naturalness, however, see [11]. For an explanation of the relationship between naturalness and renormalisability, see [12].
} 
search for QG is the presence of (particular types of) singularities in GR such as black hole singularities, and the "big bang" singularity. These are "places" where the theory is formally (mathematically) ill-defined - apparently representing a breakdown of spacetime - and QG is supposed to shed light on these.

\section{Conditions on a fundamental theory}

According to the above discussion, a fundamental theory must be:

- UV complete ("nothing beyond" formally);

- Non-perturbative (exactly solvable);

- Natural (no sensitive dependence on high-energy parameters);

- Unified;

- Single;

- Internally consistent (well-defined formally, with no problematic singularities)

Several of these ideas reflect a more general principle: That a fundamental theory not leave anything apparently in need of explanation. For instance, if a theory is not UV complete, or otherwise not well-defined everywhere, then we are led to ask what happens in the domains that the theory does not describe. More generally, if a theory is not internally consistent, or relies on approximations, physicists tend to believe that this is a symptom of there being something missing - some physics that the theory fails to take into account.

Consider if we did not have a single (i.e., lone) theory, but a "patchwork" of several (UV-complete, and otherwise apparently fundamental) theories. These would have to fit together in an especially particular way, such that there were neither any gaps, nor any overlap in the domains of the world covered by these theories. Otherwise, if there were gaps, we would ask about the phenomena not described by any of the theories, or the physics "between" the theories - we would search for a description of this. And, if there were overlap, with a particular phenomenon described by more than one theory, then we would ask which (if any) provides the more fundamental description. Thus, if the patchwork of several apparently fundamental theories did not match up perfectly, we would be led to search for a more fundamental theory.

However, if the patchwork did match up perfectly - such that, at the smallest distance scales, all physical phenomena were covered, and there was only one description of each phenomenon - we could "stitch" these all together to form a single theory. ${ }^{11}$ In this case, the theory would be single, and satisfy all conditions for fundamentality, except for unification. The idea of unification is not just that there be a single theory describing all phenomena, but that it describe all phenomena as the same - as fundamentally stemming from a single origin, e.g., as manifestations of a single entity or interaction.

\footnotetext{
${ }^{11}$ Thus, I argue that a fundamental theory should be single, contra the typical distinction drawn between a fundamental and final theory, according to which only the latter need be single (Footnote 6).
} 
The requirement of unification is hard to justify. Given that our manifest experience of the world is of diversity rather than a sameness of phenomena, seeking an explanation of heterogeneity seems counter-intuitive - surely a unified description would be more striking than a disunified one, and cry out for explanation? I return to discuss this below. For now, though, I add two more criteria to the list that are also motivated by the need for explanation. These criteria are not drawn from just from high-energy (particle) physics, however, but from other areas, including quantum mechanics and relativity. Here, it is believed that a fundamental theory must also be:

- Level comprehensive ("no gaps and no overlap" in description at the scales that the theory is required ${ }^{12}$ in order to describe);

- Background independent (no fixed structures across all models of the theory);

- "Definite" (it should be clear how the theory yields definite measurement results)

The first of these additional criteria-which I call level comprehensiveness - stems from the need for a complete and non-overlapping description of the physics at the most fundamental level. The notion of background independence has several aspects (including that the theory be non-perturbative), but the general idea is that there are no fixed ("background") structures in the theory - nothing that has to be specified for the theory "by hand" instead of being determined by the theory itself $[14,15]$. Otherwise, the appearance of such structures requires explanation, and implores us to seek a more fundamental theory that provides this.

Finally, the last requirement, "definiteness", is inspired by the measurement problem in quantum theory: According to quantum mechanics, the wave function describing a system evolves as a superposition of different states, but any measurement we make on a system always finds the system in a definite state. And, in spite of the wave function evolution being deterministic, quantum theory yields only probabilities of particular measurement outcomes. The theory does not give an answer to how it is that when we take a measurement of a something, we get a definite result. This problem is disquieting enough that many researchers seek either a more fundamental formulation of the theory, or - more drastically a more fundamental theory to solve it $[16,17,18]$. Motivated by this unease, the criterion of "definiteness" is supposed to capture the idea that a fundamental theory should have an interpretation on which we can understand both what a measurement is, as well as how the theory yields definite measurement outcomes. (This criterion could be seen as an instance of a more general requirement on a fundamental theory: That it admit of an interpretation that allows us to connect its ontology with our empirical results in a conceptually satisfying way. In other words, that it not be disjoint from manifest experience).

All nine conditions above assume a lot about the world - for instance, why should it be everywhere amenable to physical description, and why should this description be within our ability to formulate? Why should the world be such that our theories of it are formally neat and mathematically tractable, rather than messy and unusable? And, as I asked above, why does manifest diversity and disunity, rather than covert underlying unity, require explanation? Each criterion needs further justification, especially if its standardly-cited motivation

\footnotetext{
${ }^{12}$ Note that there may be overlap in lower-energy, less-fundamental descriptions.
} 
is an imagined trajectory of physics based on a particular reading of the history of physicsas, for instance, tending towards greater unification [13], or, as Weinberg [19] believes, a "convergence of the arrows of explanation".

These are all good philosophical questions for which I have no answers. What I argue, however, is that each condition - while it may not be precisely definable nor rigorously philosophically justifiable - nevertheless captures something central to the enterprise of physics itself. Physics does and must, by its nature, assume that we are able to formulate a physical description of all phenomena, and that this description is useful to us as far as it can be. If physics were to abandon this assumption, then it would seem to have "given up", in a sense: We would no longer be doing physics. It is also key to the "business of physics" that it explain diverse phenomena by appeal to simple, universal laws. It's just what physics does. And this underlies its requirement that a fundamental theory be unified.

The list of conditions has been compiled from the perspective of physics itself: It represents the criteria that any theory must satisfy if it is to be counted, by physics, as a fundamental theory. Thus, I argue that, given that this list is complete, a physical theory's fulfilment of these conditions is sufficient for that theory to be treated as fundamental according to physics. Note, however, that this list was drawn up based on current theories, and it is possible that these views change; for instance, QG may very well force us to reconsider our requirements.

Before turning to QG, however, I must emphasise that the above conditions are just those that a theory of physics - i.e., a scientific theory - must satisfy in order to be considered fundamental. In other words, I am presupposing that any candidate fundamental theory of physics already satisfies some further conditions such that it is acceptable as a scientific theory. I do not consider these further conditions (which may include requirements relating to predictions, experiment, falsifiability, etc.) - suffice to say they are even more controversial, especially given the current state of QG research.

\section{Quantum gravity}

As stated in $\S 3.2$, the motivations for QG include the desire for unification, as well as the desire for a single framework for both matter and spacetime (rather than a number of different theories describing different domains). Yet, QG it is not necessarily a unified theory, nor a theory of everything. In the first case, it may be a semiclassical theory, which is a nonunified combination of GR and QFT, and in the second case, it may just be a quantum theory of gravity, and not a theory that combines gravity with the standard model forces. In other words, although it is expected to be more-fundamental than GR and QFT, $Q G$ is not necessarily a fundamental theory [7].

Currently, there is no QG. Instead, there are a number of approaches to finding a theory (i.e., different research programs), of various stages of development, with none, as yet, yielding novel, experimentally testable predictions. One of these approaches, string theory, alleges to be a unified theory of everything. An additional claim for string theory's being a candidate for a fundamental (i.e., final) theory is its UV completeness - using a symmetry in the theory known as "T-duality", an argument can be made that string theory describes a minimal length [20]. Thus, string theory apparently says there can be no new physics 
"beyond" that described by the approach. According to string theory, then, we should stop digging.

But, as described above ( $(1)$, even if string theory is correct, its UV completeness does not alleviate the epistemic worry: It is possible that new physics exists beyond, and that a more-fundamental theory be necessary (one not featuring the minimal length described by string theory). In other words, it is possible that string theory, even if correct, turns out to be an effective theory after all.

String theorists, however, believe that this is not the case, and that the approach will produce a final theory. ${ }^{13}$ The problem, though, is that the approach does not satisfy many of the other conditions on a fundamental theory - in particular, it is only known perturbatively, it is background dependent, and its parameters are not uniquely determined (string theory is not a single theory, but a huge "landscape" of possible theories). Additionally, the approach is not level comprehensive, because of overlaps - different theories (known as dual theories) can potentially be interpreted as describing the "same physics" [21]. All of these factors drive string theorists to search for a more fundamental type of string theory underlying the currently-known "versions" of it. (Researchers in other approaches to QG, however, are duly sceptical of a positive outcome).

\section{Conclusion}

Physics progresses in a number of ways. As well as discovering increasingly general, overarching theories, it also seeks to unpeel successive layers of reality - to describe spacetime and matter at the smallest distance scales (and possibly even beyond). Attempting to understand the potential conclusion of this second endeavour, I have drafted a list of nine criteria that a fundamental theory of physics must satisfy, according to physics itself. I argued that a physical theory's fulfilment of these necessary conditions is jointly sufficient for it to be regarded, by current physics, as fundamental, while still recognising that there may be additional conditions discovered in the future. The epistemic worry that nags us to always keep digging for a more-fundamental theory - the worry that, no matter what theory we arrive at, there might still be new physics beyond - can be turned around, thanks to the two general principles that underlie the nine conditions. These principles are: Full, non-overlapping coverage of description, and comprehensiveness of explanation. If we believe these are satisfied, then the question shifts from "What if there's something beyond?" to "Why should we think there is something beyond?" That is, the burden of justification is transferred. If a theory is found that satisfies all these conditions, and yet its status as fundamental is disputed, then we can ask why. The answer will either be unacceptable, or it will reveal further conditions to add to the list. Currently, however, we are still digging.

\footnotetext{
${ }^{13}$ Arguments for this appear in [20].
} 


\section{References}

[1] Howard Georgi. Effective-field theory. Annual Review of Nuclear and Particle Science, 43:209-252, 1993.

[2] Philip W. Anderson. More is different. Science, 177:393-396, 1972.

[3] Tian Yu Cao and Silvan S. Schweber. The conceptual foundations and the philosophical aspects of renormalization theory. Synthese, 97(1):33-108, 1993.

[4] Elena Castellani. Reductionism, emergence, and effective field theories. Studies In History and Philosophy of Modern Physics, 33(2):251-267, 2002.

[5] Karen Crowther. Effective Spacetime: Understanding Emergence in Effective Field Theory and Quantum Gravity. Springer, Heidelberg, 2016.

[6] Nick Huggett and Robert Weingard. The renormalisation group and effective field theories. Synthese, 102(1):171-194, 1995.

[7] Karen Crowther and Niels Linnemann. Renormalizability, fundamentality and a final theory: The role of UV completion in the search for quantum gravity. British Journal for the Philosophy of Science, 2017.

[8] Gian F. Giudice. Naturally speaking: The naturalness criterion and physics at the LHC. In G. Kane and A. Pierce, editors, Perspectives on LHC Physics. World Scientific, 2008.

[9] James D. Wells. The utility of naturalness, and how its application to quantum electrodynamics envisages the standard model and Higgs boson. Studies in History and Philosophy of Modern Physics, 49:102-108, 2015.

[10] Porter Williams. Naturalness, the autonomy of scales, and the 125gev higgs. Studies in History and Philosophy of Science Part B: Studies in History and Philosophy of Modern Physics, 51:82 - 96, 2015.

[11] Sabine Hossenfelder. Screams for explanation: Finetuning and naturalness in the foundations of physics. arXiv:1801.02176v2, 2018.

[12] Alexander Franklin. Whence the effectiveness of effective field theories? The British Journal for the Philosophy of Science, page axy050, 2018.

[13] Tim Maudlin. On the unification of physics. Journal of Philosophy, 93(3):129-144, 1996.

[14] Gordon Belot. Background-independence. General Relativity and Gravitation, 43(10):2865-2884, 2011.

[15] Lee Smolin. The case for background independence. In D. Rickles, S. French, and J. Saatsi, editors, The Structural Foundations of Quantum Gravity, pages 196-239. Oxford University Press, Oxford, 2006.

[16] David Albert. Quantum Mechanics and Experience. Harvard University Press, Cambridge, 1992.

[17] Roland Omnès. Understanding Quantum Mechanics. Princeton University Press, Princeton, 1999.

[18] Simon Saunders, Jonathan Barrett, Adrian Kent, and David Wallace, editors. Many Worlds? Everett, Quantum Theory Reality. Oxford University Press, Oxford, 2010.

[19] Steven Weinberg. Newtonianism, reductionism and the art of congressional testimony. Nature, (330):433-437, 1987.

[20] Richard Dawid. String Theory and the Scientific Method. Cambridge University Press, 2013.

[21] Elena Castellani and Dean Rickles, editors. Special Issue on Dualities in Physics. Studies in History and Philosophy of Modern Physics, 59:1-142, 2017. 